\title{
Avaliação da independência funcional e da qualidade de vida após a alta da unidade de terapia intensiva: um estudo do coorte prospectivo
}

\section{Evaluation of functional independence and quality of life after discharge of intensive care unit: a prospective cohort study}

\section{André Luiz Cordeiro' 1 \\ Francielle Peixoto ${ }^{2}$ (]) \\ Maya Menezes ${ }^{3}$ (1) \\ Franciele Norberto ${ }^{4}$ (1)}

\section{Fernanda Machado Kutchak 5 Marcelo Rieder ${ }^{6}$ Bruno Prata Martinez ${ }^{7}$ Luiz Alberto Forgiarini Junior ${ }^{8}$ (1)}

1Autor para correspondência. Centro Universitário Nobre (Feira de Santana). Bahia, Brasil. andrelisboacordeiro@gmail.com ${ }^{2-4,6}$ Centro Universitário Metodista (Porto Alegre). Rio Grande do Sul, Brasil. francielepeixoto@gmail.com, mayamenezes@gmail.com, francielenorberto@gmail.com, marcelorieder@gmail.com ${ }^{5}$ Hospital Cristo Redentor (Porto Alegre). Rio Grande do Sul, Brasil. fernandakutchak@gmail.com 7Universidade Federal da Bahia (Salvador). Bahia, Brasil. brunopmartinez@gmail.com ¿Universidade La Salle (Canoas). Rio Grande do Sul, Brasil. forgiarini.luiz@gmail.com

RESUMO | INTRODUÇÃO: Os pacientes criticamente doentes podem sofrer alterações funcionais, sociais e mentais, incluindo deficiência de conhecimento, memória e concentração após a admissão na unidade de terapia intensiva (UTI) e alta hospitalar. Apesar do conhecimento sobre o impacto da internação hospitalar na funcionalidade e qualidade de vida, ainda há pouco reconhecimento na literatura sobre este impacto a longo prazo. OBJETIVO: Verificar a independência funcional e a qualidade de vida (QOL) em pacientes acima de 1 ano após a alta da Unidade de Tratamento Intensivo (UTI). MÉTODOS: Este é um estudo de coorte prospectivo, foram admitidos na UTI e sob ventilação mecânica por mais de 48 horas, até 24 horas após a alta da UTI, acima de 18 anos de idade, de ambos os sexos e que concordaram em participar do estudo. Foram excluídos aqueles com sequelas neurológicas e traumas recorrentes que impossibilitavam a avaliação funcional. As variáveis independência funcional (Medida de Independência Funcional - FIM) e QOL foram medidas utilizando o questionário do formulário curto 36 (SF-36) no momento da alta da UTI (período I), 30 dias (período II), e 1 ano após (período III). RESULTADOS: 33 pacientes foram incluídos no estudo com uma idade média de $49,13 \pm 16,3$ anos, e a principal causa de hospitalização foi distúrbios neurológicos, dos quais 5(14,70\%) morreram, e dois não completaram as avaliações, resultando em um total de 26 pacientes avaliados 1 ano após a alta. A duração da internação na UTI (dias) foi de 16 (7 - 22) e o tempo de ventilação mecânica (dias) foi de 8,5 (2 - 13). Dez pacientes lá foram diagnosticados com sepse. Foram encontrados os seguintes valores nesses três momentos para as variáveis IF (I-51 [47-64,5]; II-80[59,5-108]; III104[82,8-123]) e QOL(I-67,4[57,3-81,1]; II-80,2[70,1-99,2]; III-93,5[88,5-96,5]). A independência funcional e QOL aumentou significativamente entre os momentos II e III, em comparação com os momentos I ( $p<0,05)$, sem diferença na comparação do período II em relação ao III. CONCLUSÃO: Os pacientes recuperam sua funcionalidade e qualidade de vida após trinta dias de alta da UTI. Entretanto, a funcionalidade e a qualidade de vida deste paciente não mudam após 1 ano de alta da UTI em relação ao período de 30 dias após a alta da UTI.

PALAVRAS-CHAVE: Funcionalidade. Unidade de Terapia Intensiva. Qualidade de Vida. Fisioterapia.
ABSTRACT | INTRODUCTION: Critically ill patients may experience functional, social, and mental changes, including impaired cognition, memory, and concentration after admission to the intensive care unit (ICU) and hospital discharge. Despite the knowledge about the impact of the hospital stay on functionality and quality of life, there is still little recognition in the literature of this impact in the long term. OBJECTIVE: To verify functional independence and quality of life (QOL) in patients over 1 year after discharge from the Intensive Care Unit (ICU). METHODS: This is a prospective cohort study, were admitted to the ICU and under mechanical ventilation for more than 48 hours, up to 24 hours after discharge from the ICU, over 18 years of age, of both sexes and who agreed to participate in the study. Those with neurological sequelae and recurrent trauma that made functional assessment impossible were excluded. Functional independence (Functional Independence Measure-FIM) and QOL variables were measured using the Short Form 36 questionnaire (SF-36) at the time of discharge from the ICU (period I), 30 days (period II), and 1 year after (period III). RESULTS: 33 patients were included in the study with a mean age of $49.13 \pm 16.3$, and the main cause of hospitalization was neurological disorders, of which $5(14.70 \%)$ died, and two did not complete the evaluations, resulting in a total of 26 patients evaluated 1 year after discharge. The length of ICU stay (days) was 16 (7 - 22) and the Mechanical Ventilation time (days) was 8,5 (2 - 13). Ten patients there were diagnosed with sepsis. The following values over these three moments were found for the variables IF (I-51 [47-64.5]; II-80[59.5-108]; III-104[82.8-123]) and QOL(I-67.4[57.3-81.1]; II-80.2[70.1-99.2]; III-93.5[88.596.5]). Functional independence and QOL increased significantly between moments II and III compared to moments I ( $p<0.05$ ), with no difference, when comparing period II in relation to III. CONCLUSION: Patients recover their functionality and quality of life after thirty days of discharge from the ICU. However, this patient's functionality and quality of life do not change after 1 year of discharge from the ICU in relation to the period of 30 days after discharge from the ICU.

KEYWORDS: Functionality. Intensive Care Unit. Quality of Life. Physiotherapy. 


\section{Introdução}

Pacientes críticos podem apresentar alterações funcionais, sociais e mentais, incluindo comprometimento da cognição, memória e concentração após a admissão na Unidade de Terapia Intensiva (UTI) e alta hospitalar. ${ }^{1}$ A recuperação costuma ser lenta, prolongada e as alterações podem persistir após a internação. Frequentemente, os pacientes necessitam de suporte contínuo de familiares e cuidadores após o retorno para casa, principalmente aqueles ventilados mecanicamente durante a permanência na UTI.,$\frac{2,3}{3}$ Além de todas as mudanças relacionadas à permanência na UTI, o aumento da sobrevida é acompanhado por impacto na qualidade de vida (QV) em curto e longo prazo após a alta. ${ }^{4}$

A aplicação de escalas de funcionalidade para avaliação de pacientes críticos é de extrema importância para o fisioterapeuta, pois tem como principal objetivo minimizar a perda funcional adquirida, além de preservar a capacidade de manter as habilidades necessárias para a realização das atividades de vida diária (AVDs), com ênfase na transferência e locomoção. $\frac{5}{A}$ avaliação do desempenho funcional é cada vez mais vista como uma medida valiosa de resultados de testes clínicos e é comumente utilizada com a finalidade de identificar o diagnóstico, prognóstico e comparar a resposta ao tratamento dos pacientes, bem como verificar e monitorar o desempenho da funcionalidade para orientar o terapeuta na elaboração de tratamentos e prevenção de deficiências físicas.,, 7 Uma das variáveis que promovem impacto na funcionalidade é a força muscular global, que pode ser mensurada através da força de preensão palmar.

Sabe-se que a independência funcional na alta pode estar relacionada à pior qualidade de vida e mortalidade após a alta hospitalar e o monitoramento dessas variáveis no pós-alta é de suma importância para melhor compreensão desses desfechos. ${ }^{8.9}$ Apesar do conhecimento sobre o impacto da internação na funcionalidade e qualidade de vida, ainda há pouco reconhecimento na literatura desse impacto em longo prazo. Assim, o presente estudo teve como objetivo avaliar a variação da independência funcional e qualidade de vida ao longo de 1 ano após a alta da UTI, comparando os momentos de alta imediata da UTI, trinta dias e 1 ano após a alta da UTI. Como objetivo secundário, foi avaliada a força de preensão palmar para identificar a fraqueza muscular no momento da alta da UTI.

\section{Métodos}

Estudo de coorte prospectivo realizado na UTI do Hospital Cristo Redentor e enfermarias do próprio hospital, pertencente ao Grupo Hospitalar Conceição, na cidade de Porto Alegre. As avaliações foram realizadas no período de março de 2014 a setembro de 2016. O estudo foi aprovado pelos dois Comitês de Ética em Pesquisa (parecer $n^{\circ}$ 866.267) e todos os pacientes assinaram o Termo de Consentimento Livre e Esclarecido (TCLE).

\section{Critério de elegibilidade}

O estudo incluiu pacientes que estiveram internados na UTI e sob ventilação mecânica por mais de 48 horas, que estiveram até 24 horas após a alta da UTI, maiores de 18 anos, de ambos os sexos e que aceitaram participar do estudo. Foram excluídos os pacientes com alterações neurológicas e traumatológicas prévias à admissão. Foram considerados perdas os pacientes que faleceram antes de 30 dias ou foram transferidos para outro hospital. Participaram da pesquisa os pacientes que receberam alta da UTI nas primeiras 24 horas após a saída, já no leito do paciente, 30 dias após a alta e 1 ano após a alta.

\section{Protocolo do estudo}

Os pacientes que receberam alta da UTI foram convidados a participar do estudo (no momento da alta ou quando estavam até 24 horas após a alta na enfermaria), após a aceitação e assinatura do TCLE, a ficha de avaliação foi preenchida e os dados de identificação, diagnóstico clínico, tempo de permanência na UTI, tempo de VM e se houve diagnóstico de sepse durante o período de internação. 
Após ser avaliada a independência funcional por meio da Medida de Independência Funcional (MIF) ${ }^{10}$ e a qualidade de vida foi verificada pelo Short-Form 36 (SF-36) ${ }^{11}$ e força de preensão manual. ${ }^{12}$ As entrevistas para avaliação da qualidade de vida, MIF no período de 30 dias e um ano após a alta foram realizadas por contato telefônico. Ressalta-se que as avaliações em todos os momentos foram realizadas por um examinador cego.

\section{Instrumentos de coleta}

\section{Medida de Independência Funcional}

A Medida de Independência Funcional que visa mensurar o que a pessoa realmente realiza, independente do diagnóstico, gerando escore válido para limitação ou não. Essa escala avalia a capacidade do paciente em desenvolver cuidados com o corpo, controle esfincteriano, transferência e locomoção, além de funções cognitivas como comunicação e memória. É atribuída uma pontuação de 1 a 7 , sendo que o menor valor correspondeu ao paciente totalmente dependente e o valor máximo foi aquele paciente completamente independente do ponto de vista funcional, atingindo um valor máximo de 126 pontos quando todas as variáveis foram somadas..$^{10}$

\section{Qualidade de Vida}

O Medical Outcomes Short-Form Health Survey (SF-36) é um instrumento genérico de avaliação de saúde, originalmente criado em inglês, de fácil administração e compreensão. É composto por 36 questões, abrangendo oito componentes, capacidade funcional, aspectos físicos, dor, estado geral de saúde, vitalidade, aspectos sociais, aspectos emocionais e saúde mental avaliados por 35 questões e mais uma questão comparativa entre atual e saúde há um ano.11

\section{Preensão palmar}

A manobra utilizada para medir a força por meio de um dinamômetro estava de acordo com as recomendações da American Society of Hand Therapists, que preconiza que o sujeito esteja sentado com o ombro aduzido e rodado de forma neutra, cotovelo fletido a $90^{\circ}$, antebraço em posição neutra e o punho entre $0^{\circ}$ e $30^{\circ}$ de extensão e $0^{\circ}$ a $15^{\circ}$ de desvio ulnar. 12

\section{Análise Estatística}

As variáveis categóricas foram expressas em valores absolutos e percentuais, enquanto as variáveis contínuas foram descritas por meio de médias e desvios-padrão ou medianas e intervalos interquartis. A normalidade dos dados foi avaliada pelo teste de Shapiro-Wilk. Para comparar os diferentes períodos de estudo, utilizou-se o teste de Kruskal-Wallis com o Post-hoc de Dunn. As análises foram realizadas por meio do programa Statistical Package for Social Science 20.0 (SPSS).

\section{Resultados}

Durante o período estabelecido para a coleta de dados, 33 pacientes foram incluídos no estudo e durante o estudo $5(14,7 \%)$ pacientes morreram, dois pacientes não responderam a novas avaliações, totalizando o número de pacientes avaliados para 26 em um ano após a alta do hospital. na UTI (Figura 1). 


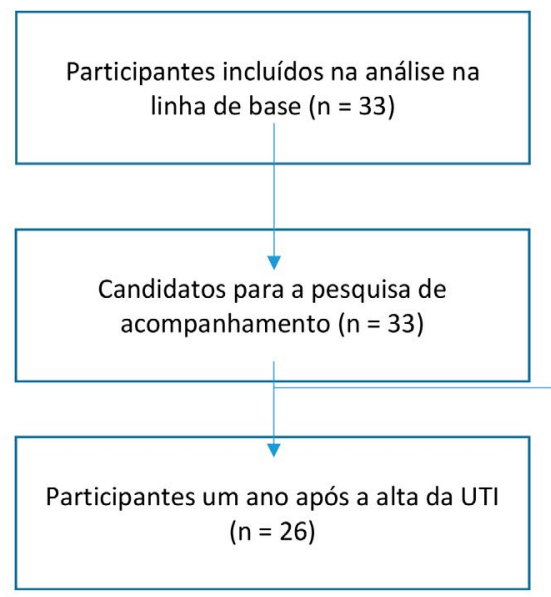

Indivíduos excluídos $(\mathrm{n}=7)$

Faleceu $(n=5)$

Não responder a novas avaliações $(n=2)$

Os pacientes tinham média de idade de 49,13 $\pm 16,3$ anos, com predomínio do sexo feminino e a condição neurológica como principal causa de internação, conforme tabela 1.

Tabela 1. Características dos pacientes incluídos no estudo $(n=33)$

\begin{tabular}{ll}
\hline Variável & Resultados \\
\hline Idade (anos) & $49,13 \pm 16,3$ \\
\hline Tempo Total de Hospitalização (dias) & $36,76 \pm 12,70$ \\
\hline Tempo de permanência na UTI (dias) & $16(7-22)$ \\
\hline Tempo de VM (dias) & $8,5(2-13)$ \\
\hline Número de sessões fisioterapêuticas na UTI & \multicolumn{1}{c}{$39(30-48)$} \\
\hline Gênero & \\
Feminino & $18(56,25)$ \\
Masculino & $14(43,75)$ \\
\hline Causas de Hospitalização & $14(43,76)$ \\
Neurológica & $9(28,12)$ \\
Politrauma & $9(28,12)$ \\
Outros & $10(31,25)$ \\
\hline Sepse & $5(15,62)$ \\
\hline Trombose Venosa Profunda & \\
\hline
\end{tabular}

Dados expressos em média +/- desvio padrão em (\%). UTI: unidade de terapia intensiva; VM: ventilação mecânica.

A avaliação da força muscular periférica na alta da UTI mostrou valores de 13,5 \pm 8,2 kg para o membro dominante. Ao analisar a MIF, observamos que 30 dias após a alta da UTI, o único item que não apresentou melhora significativa foi a comunicação, que apresentou melhora um ano após a alta quando comparada com a alta da UTI, não havendo diferença nos 30- comparação de dias após a alta em um ano.

A análise da qualidade de vida mostrou que mesmo havendo melhora no valor total entre a alta da UTI e trinta dias e um ano depois, observou-se que alguns fatores não aumentaram significativamente (Tabela 2). 
Tabela 2. Avaliação da escala MIF e qualidade de vida no momento da alta da UTI e 30 dias após e um ano após ( $\mathrm{n}=33$ )

\begin{tabular}{llll}
\hline Variável & Alta da UTI & Após 30 dias & After one year \\
\hline Medida de Independência Funcional & & & $34,48 \pm 17,04^{\mathrm{b}}$ \\
Cuidados pessoais & $14,28 \pm 6,0$ & $24,40 \pm 10,6^{\mathrm{a}}$ & $12 \pm 3,4^{\mathrm{b}}$ \\
Controle de esfincter & $9,22 \pm 4,4$ & $11,90 \pm 3,6^{\mathrm{a}}$ & $14,96 \pm 4,8^{\mathrm{b}}$ \\
Mobilidade & $5,50 \pm 3,1$ & $11,53 \pm 6,2^{\mathrm{a}}$ & $7,72 \pm 3,38^{\mathrm{b}}$ \\
Locomoção & $2,94 \pm 1,7$ & $5,97 \pm 4,0^{\mathrm{a}}$ & $18,56 \pm 7,16^{\mathrm{b}}$ \\
Comunicação & $12,25 \pm 5,8$ & $14,60 \pm 5,9$ & $16,44 \pm 4,08^{\mathrm{b}}$ \\
Cognição social & $9,72 \pm 5,7$ & $13,23 \pm 5,5^{\mathrm{a}}$ & $104(82,8-123)^{\mathrm{b}}$ \\
Total & $51(47-64,5)$ & $80(59,5-108)^{\text {a }}$ & \\
Qualidade de Vida (SF-36) & & & $16,5 \pm 5,63$ \\
Capacidade funcional & $3,75 \pm 6,1$ & $26,17 \pm 23,9^{\mathrm{a}}$ & $5,86 \pm 1,42$ \\
Limitação por aspectos físicos & $11,72 \pm 25,0$ & $15 \pm 20,0$ & $7,68 \pm 2,47$ \\
Dor & $14,66 \pm 17,7$ & $41,23 \pm 26,1^{\text {a }}$ & $13,31 \pm 3,19$ \\
Estado geral da saúde & $43,63 \pm 22,3$ & $40,53 \pm 22,0^{\circ}$ & $12,81 \pm 3,40$ \\
Vitalidade & $26,72 \pm 20,9$ & $36,83 \pm 24,1$ & $6,40 \pm 1,61$ \\
Aspectos sociais & $21,88 \pm 24,0$ & $38,75 \pm 24,0^{\text {a }}$ & $4,27 \pm 1,28$ \\
Limitação por aspectos emocionais & $13,46 \pm 29,8$ & $16,65 \pm 23,9$ & $20 \pm 4,23$ \\
Saúde mental & $47,94 \pm 22,6$ & $63,47 \pm 23,1^{\text {a }}$ & $93,5(88,5-96,5)^{\mathrm{b}}$ \\
Total & $67,4(57,3-81,1)$ & $80,2(70,1-99,2)^{\mathrm{a}}$ & \\
\hline
\end{tabular}

Dados expressos em média \pm desvio padrão e mediana (mínimo - máximo). a p <0,05 30 dias vs. alta da UTI. b p <0,05 1 ano vs. alta da UTI.

\section{Discussão}

No presente estudo, observamos a melhora funcional e da qualidade de vida dos pacientes trinta dias e um ano após a alta da UTI. Isso não está relacionado ao tempo de internação, ventilação mecânica ou presença de sepse em comparação à alta imediata da UTI. Demonstramos também que não há diferença na qualidade de vida e funcionalidade quando comparamos o período de 30 dias e um após a alta. Após trinta dias, variáveis como autocuidado, controle esfincteriano, mobilidade, locomoção e cognição social foram significativas quando comparadas ao tempo de alta hospitalar. Todos continuaram a ter significado após um ano, com a adição da comunicação. Em termos de qualidade de vida, Capacidade Funcional, Dor, Aspectos Sociais e Saúde Mental foram significativos quando comparados aos elevados na UTI.

Ao avaliar a funcionalidade através das escalas MIF e Barthel na qualidade de vida através do SF-36, demonstramos a melhora em ambas as condições no período de 30 dias e um ano após a alta da UTI. Van der Schaaf et al. ${ }^{13}$, em estudo de coorte que avaliou 116 pacientes ventilados mecanicamente por mais de 48 horas em um período de 3, 6 e 12 meses. Os autores utilizaram o método Sickness Impact Profile e demonstraram que 1 ano após a alta da UTI, cerca de $69 \%$ dos pacientes ainda apresentam restrições em suas AVDs e que apenas $50 \%$ dos pacientes retornaram às atividades relacionadas ao trabalho. De acordo com nosso estudo, houve melhora significativa da independência funcional e da qualidade de vida após um ano, o que discorda em partes dos autores citados. Essa divergência pode residir no instrumento de avaliação utilizado e no tamanho da amostra, e erro estatístico do tipo I pode ter ocorrido no presente estudo. 
Outro estudo, que avaliou pacientes em VM por tempo prolongado, demonstrou que após a alta hospitalar, apenas $9 \%$ deles obtiveram resultados positivos em relação à independência funcional social e cognitiva. ${ }^{14}$ Segundo os autores, esse fato pode estar relacionado ao tempo de ventilação mecânica, que causa complicações sistêmicas como mecânica pulmonar, muscular periférica e de múltiplos órgãos, causando grande morbidade a esses pacientes. $O$ impacto negativo é alto, podendo expor os pacientes a níveis inadequados de assistência, resultando em óbitos inesperados ou reinternações na UTI durante a mesma internação. $\frac{15}{}$ Nosso estudo constatou que trinta dias após a alta hospitalar, houve mudanças na cognição e nos aspectos sociais, mas após um ano essa mudança foi menos sensível. Isso pode ser explicado pelo perfil do paciente incluído nesta pesquisa e pela falta de compreensão do nível de atividade física realizado por eles após a alta hospitalar.

Dettling-Ihnenfeldt et al., sobre a funcionalidade, nos mostram o quanto a mobilização precoce desses pacientes melhora a longo prazo. Em uma análise de 133 pacientes avaliados durante 3, 6 e 12 meses após a alta, eles apresentaram estado de saúde funcional prejudicado aos 3 meses após a alta da UTI, não aos 6 e 12 meses. $\frac{16}{16} \mathrm{Um}$ dos motivos que podem modificar o resultado funcional dos pacientes internados na UTI é a realização da fisioterapia. Tem como objetivo promover a recuperação e preservação da funcionalidade, resultando na redução do tempo de desmame, ventilação mecânica e hospitalização. ${ }^{17,18}$ Assim, a fisioterapia ajuda a melhorar a funcionalidade e a qualidade de vida pós-alta.

No decorrer de nosso estudo, não mostramos correlação entre o número de consultas e os desfechos avaliados. A literatura mostra que pacientes diagnosticados com fraqueza muscular adquirida na UTI (FMA-ICU), apresentam pior prognóstico funcional, no período de 3 a 12 meses, após a alta. ${ }^{14,19}$

Em outro estudo sobre funcionalidade e mortalidade, os pacientes apresentaram comprometimento do estado funcional significativamente maior na alta hospitalar do que os pacientes com menor tempo de internação na UTI, e essa diferença persistiu após três meses. ${ }^{20}$ Os níveis de independência no seguimento de um ano nunca foram semelhantes ao basal, corroborando com nosso estudo onde a variável comunicação MIF aumentou em um ano, em contraste com a variável capacidade funcional do questionário SF36, que diminuiu consideravelmente. Uma possível alternativa para essa população de pacientes críticos após a alta da UTI poderia ser um programa de reabilitação multicomponente combinando treinamento cognitivo, físico e funcional, podendo assim melhorar o desempenho cognitivo e melhorar os resultados funcionais. $\underline{\underline{21}}$

O presente estudo tem como limitações o pequeno tamanho da amostra e a ausência de avaliação da gravidade no momento da admissão na UTI. Outra limitação foi a coleta da medida de independência funcional com base no autorrelato, o que pode levar a viés de aferição, bem como o não controle das intervenções realizadas no ambiente domiciliar, o que pode impactar nos desfechos avaliados.

\section{Conclusão}

Os pacientes recuperam sua funcionalidade e qualidade de vida após trinta dias da alta da UTI, porém a funcionalidade e qualidade de vida desse paciente não se altera após 1 ano da alta da UTI em relação ao período de 30 dias após a alta da UTI.

\section{Contribuição dos autores}

Cordeiro ALL participou da análise e interpretação dos dados, redação do manuscrito e revisão crítica do manuscrito quanto ao conteúdo intelectual. Peixoto $F$, Menezes $M$ e Norberto $F$ participaram da concepção e desenho da pesquisa, obtenção de dados, análise e interpretação dos dados e redação do manuscrito. Kutchak FM e Rieder M participaram da concepção e desenho da pesquisa, obtenção de dados, análise e interpretação dos dados, redação do manuscrito e revisão crítica do manuscrito quanto ao conteúdo intelectual importante. Martinez BP participou da redação do manuscrito e revisão crítica do manuscrito quanto ao conteúdo intelectual. Forgiarini Junior LA participou da concepção e desenho da pesquisa, obtenção de dados, análise e interpretação dos dados, redação do manuscrito e revisão crítica do manuscrito quanto ao conteúdo intelectual.

\section{Conflito de interesses}

Nenhum conflito financeiro, legal ou político envolvendo terceiros (governo, corporações e fundações privadas etc.) do manuscrito, análise estatística, etc.). 


\section{Referências}

1. Slooter AJC, Van De Leur RR, Zaal IJ. Delirium in critically ill patients. Handb Clin Neurol. 2017;141:449-66. https://doi. org/10.1016/B978-0-444-63599-0.00025-9

2. Rengel KF, Hayhurst CJ, Pandharipande PP, Hughes CG. Longterm cognitive and functional impairments after critical illness. Anesth Analg. 2019;128(4):772-80. https://doi.org/10.1213/ ANE.0000000000004066

3. Valko L, Baglyas S, Gyarmathy VA, Gal J, Lorx A. Home mechanical ventilation: quality of life patterns after six months of treatment. BMC Pulm Med. 2020;20(1):221. https://doi. org/10.1186/s12890-020-01262-Z

4. Mafra JMES, Silva JM, Silveira LTY, Fu C, Tanaka C. Quality of life of critically ill patients in a developing country: a prospective longitudinal study. Phys Ther Sci. 2016;28(10):2915-20. https://doi. org/10.1589/jpts.28.2915

5. Hermans G, Van den Berghe G. Clinical review: intensive care unit acquired weakness. Crit Care. 2015;19(1):274. https://doi. org/10.1186/s13054-015-0993-7

6. Zhang L, Hu W, Cai Z, Liu J, Wu J, Deng Y, et al. Early mobilization of critically ill patients in the intensive care unit: A systematic review and meta-analysis. PLoS One. 2019;14(10):e0223185. https://doi.org/10.1371/journal.pone.0223185

7. Zang K, Chen B, Wang M, Chen D, Hui L, Guo S, et al. The effect of early mobilization in critically ill patients: A meta-analysis. Nurs Crit Care. 2020;25(6):360-7. https://doi.org/10.1111/nicc.12455

8. Hu Y, Hu X, Xiao J, Li D. Effect of early mobilization on the physical function of patients in intensive care unit: a Meta-analysis [Internet]. Zhonghua Wei Zhong Bing Ji Jiu Yi Xue. 2019;31(4):45863. Disponível em: https://europepmc.org/article/med/31109421

9. Connolly B, Salisbury L, O'Neill B, Geneen L, Douiri A, Grocott $M P W$, et al. Exercise rehabilitation following intensive care unit discharge for recovery from critical illness: executive summary of a Cochrane Collaboration systematic review. J Cachexia Sarcopenia Muscle. 2016;7(5):520-26. https://doi.org/10.1002/ jcsm.12146

10. Oliveira AB, Martinez BP, Gomes Neto MG. Impacto do internamento em uma unidade de terapia intensiva na independência funcional. Rev Bras Ter Intensiva [Internet]. 2010;(suppl S95). Disponível em: https://rbti.org.br/exportarsuplemento/RBTI_Suplemento_2010.pdf

11. Jenkinson C, Coulter A, Wright L. Short form 36 (SF36) health survey questionnaire: normative data for adults of working age. BMJ. 1993;306(6890):1437-40. https://doi.org/10.1136/ bmj.306.6890.1437
12. Moreira D, Álvarez RRA, Gogoy JR, Cambraia AN. Abordagem sobre preensão palmar utilizando o dinamômetro JAMARß: uma revisão de literatura [Internet]. Rev Bras Ciênc Mov. 2003;11(2):959. Disponível em: https://pesquisa.bvsalud.org/portal/resource/ pt/lil-524709

13. Van der Schaaf M, Beelen A, Dongelmans DA, Vroom MB, Nollet $F$. Functional status after intensive care: a challenge for rehabilitation professionals to improve outcome. J Rehabil Med. 2009;41(5):360-6. https://doi.org/10.2340/16501977-0333

14. Curzel J, Forgiarini Júnior LA, Rieder MM. Evaluation of functional independence after discharge from the intensive care unit. Rev Bras Ter Intensiva. 2013;25(2):93-8. Citado em: PMID: 23917973

15. Pintado MC, Villa P, Luján J, Trascasa M, Molina R, GonzálezGarcía N, et al. Mortality and functional status at one-year of follow-up in elderly patients with prolonged ICU stay. Med Intensiva. 2016;40(5):289-97. https://doi.org/10.1016/j. medin.2015.08.002

16. Dettling-Ihnenfeldt DS, Wieske L, Horn J, Nollet F, Van der Schaaf M. Functional recovery in patients with and without intensive care unit-acquired weakness. Am J Phys Med Rehabil. 2017;96(4):236-42. https://doi.org/10.1097/ PHM.0000000000000586

17. Alaparthi GK, Gatty A, Samuel SR, Amaravadi SK. Effectiveness, Safety, and Barriers to Early Mobilization in the Intensive Care Unit. Crit Care Res Pract. 2020(2020):7840743. https://doi. org/10.1155/2020/7840743

18. Lai CC, Chou W, Chan KS, Cheng KC, Yuan KS, Chao CM, et al. Early mobilization reduces duration of mechanical ventilation and intensive care unit stay in patients with acute respiratory failure. Arch Phys Med Rehabil. 2017;98(5):931-39. https://doi. org/10.1016/j.apmr.2016.11.007

19. Secombe PJ, Stewart PC. Long-term morbidity and mortality in survivors of critical illness: a 5-year observational follow-up study. Rural Remote Health. 2017;17(1):3908. https://doi.org/10.22605/ $\underline{\text { rrh3908 }}$

20. Araujo TG, Rieder MM, Kutchak FM, Franco Filho JW. Readmissions and deaths following ICU discharge: a challenge for intensive care. Rev Bras Ter Intensiva. 2013;25(1):32-8. https://doi. org/10.1590/S0103-507X2013000100007

21. Jackson JC, Ely EW, Morey MC, Anderson VM, Denne LB, Clune $J$, et al. Cognitive and physical rehabilitation of intensive care unit survivors: results of the RETURN randomized controlled pilot investigation. Crit Care Med. 2012;40(4):1088-97. https://doi. org/10.1097/CCM.0b013e3182373115 